\title{
Analysis of dynamic properties of alternative sequences to the Petlyuk column
}

\author{
Juan Gabriel Segovia-Hernández ${ }^{\mathrm{a}, \mathrm{b}}$, Salvador Hernández ${ }^{\mathrm{b}}$, Arturo Jiménez ${ }^{\mathrm{a}, *}$ \\ ${ }^{a}$ Instituto Tecnológico de Celaya, Departamento de Ingeniería Química, Av. Tecnológico y García Cubas s/n, Celaya, Gto. 38010, México \\ ${ }^{\mathrm{b}}$ Universidad de Guanajuato, Facultad de Química, Noria Alta s/n, Guanajuato, Gto. 36050, México
}

\begin{abstract}
The Petlyuk distillation configuration has received considerable attention because of its efficiency to reduce the energy required for the separation of ternary mixtures. The structure of the Petlyuk system offers some control challenges arising from the transfer of vapor streams back and forth between the columns. This means that neither of the columns can have a uniform lower nor higher pressure. Recently, some alternate Petlyuk-like schemes that might provide better operational properties than the Petlyuk column have been proposed. In this work, we analyze the control properties of six alternative thermally coupled distillation schemes to the Petlyuk system. The theoretical control properties are analyzed with the application of the singular value decomposition technique. Rigorous closed loop simulations are used to supplement the theoretical analysis. The results indicate that a reduction in the number of interconnections of the Petlyuk configuration does not necessarily provide an improvement of its controllability properties.
\end{abstract}

(C) 2005 Published by Elsevier Ltd.

Keywords: Thermally coupled distillation; Petlyuk column; Distillation control

\section{Introduction}

Distillation is the most widely used separation method in the chemical process industry and is the largest energy consumer among process units. This fact has provided a continuous incentive towards the search for more energy-efficiency distillation systems. One of the options to design nonconventional distillation schemes with better energy efficiencies is the use of thermal coupling. A thermally coupled structure shows an interconnection between two columns with a liquid or vapor extraction from the first column and a recycle stream from the other column in the other phase; such interconnection can be implemented in place of a reboiler or a condenser of one of the columns. A good number of works has been reported for the separation of ternary mixtures, with three thermally coupled systems gaining special interest, namely the system with a side rectifier (TCDS-SR; Fig. 1a), the scheme with a side stripper (TCDS-SS; Fig. 1b),

\footnotetext{
* Corresponding author.

E-mail address: arturo@iqcelaya.itc.mx (A. Jiménez).
}

and the fully thermally coupled distillation system (or Petlyuk column, Fig. 1c). Lower energy consumption levels or lower yearly costs of thermally coupled systems with respect to conventional sequences have been reported (e.g. Fidkowski \& Krolikowski, 1990; Glinos \& Malone, 1988; Tedder \& Rudd, 1978; Yeomans \& Grossmann, 2000). Also, those three thermally coupled systems, over a wide range of relative volatilities and feed compositions, have been reported to provide a better thermodynamic efficiency than the conventional distillation configurations (Flores, Cárdenas, Hernández, \& RicoRamírez, 2003). When comparing the energy savings among the thermally coupled schemes, it has been found that in general the Petlyuk system offers better savings than the systems with side columns. Despite the incentive on energy and equipment savings provided by thermally coupled schemes, their industrial implementation has been constrained because of the potential control problems associated with the operation of a more complex configuration (Dünnebier \& Pantelides, 1999). Nonetheless, some works have been reported on the dynamic properties of thermally coupled systems that have shown a rather promising perspective on the operational char- 


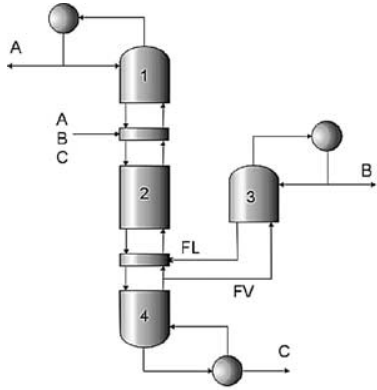

(a) TCDS- SR

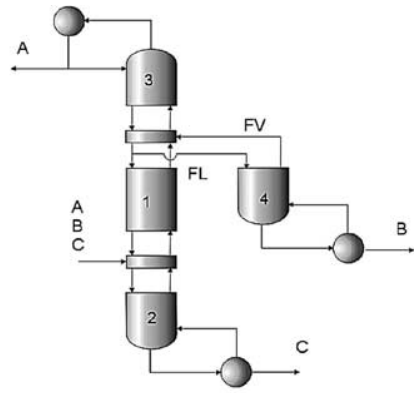

(b) TCDS- SS

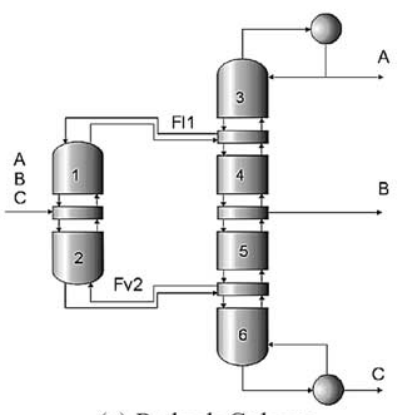

(c) Petlyuk Column

Fig. 1. Thermally coupled distillation sequences for the separation of ternary mixtures.

acteristics of such systems (Abdul Mutalib \& Smith, 1998; Hernández \& Jiménez, 1999a; Jiménez, Hernández, Montoy, \& Zavala-García, 2001; Segovia-Hernández, Hernández, \& Jiménez, 2002; Segovia-Hernández, Hernández, RicoRamírez, \& Jiménez, 2004; Wolff \& Skogestad, 1995).

Some alternative options and extensions to the systems shown in Fig. 1 have been suggested (Agrawal \& Fidkowski, 1998, 1999; Rong \& Kraslawski, 2003). Some of those structures aim for some type of simplification to the configuration of the original thermally coupled systems of Fig. 1, with the expectation of improving the controllability properties of the original systems. In particular, the Petlyuk column provides a fully interconnected structure, with two thermal couplings that result in vapor interconnections flowing back and forth between the columns. This poses a design challenge on the system, since neither column can be designed at a uniformly higher pressure than the other one. Since the Petlyuk structure typically provides the highest energy savings from the systems of Fig. 1, a special interest to provide modified configurations to such arrangement has arisen. As a result, several modifications on its structure have been proposed, some of them providing simpler designs that might be more operable than the original Petlyuk configuration, for instance, through the elimination of one of the thermal couplings. Some of the newer structures that have been suggested to improve the expected control properties of the Petlyuk system have recently been analyzed in terms of their potential energy requirements, and found in many cases to provide similar energy savings as the original system (Jiménez, Ramírez, Castro, \& Hernández, 2003). Such results provide an incentive for the analysis of the dynamic properties of the new designs to see how they may improve the controllability properties of the Petlyuk configuration. In this work, we conduct an initial analysis on the theoretical control properties of six alternative schemes to the Petlyuk column and compare them to those of the original configuration. The analysis is carried out through the application of the singular value decomposition technique, followed by a set of dynamic tests under feedback operation.

\section{Alternative schemes to the Petlyuk distillation configuration}

The Petlyuk system (Fig. 1c) shows a bidirectional flow of the interconnecting vapor streams. This situation would require that the pressure at the bottom of the prefractionator be lower than that of the main column, while the pressure at the top should be higher than that of the main column. This aspect might provide a major concern for its proper implementation and operation. Agrawal and Fidkowski (1998, 1999) have addressed this problem and suggested some conceptual modifications to the Petlyuk configuration that might improve its dynamic and operational properties. One way to do this is to use unidirectional flows between the columns. Another way is to reduce the number of interconnections, thus providing simpler arrangements (and therefore systems with better control perspectives.) Fig. 2 shows six alternative structures to the Petlyuk column that, in principle, could provide more operable systems. The topology of the new designs follows directly from the original arrangement, since the six sections of the Petlyuk system depicted in Fig. 1c provide the basis for the structures of the new systems; the corresponding sections are identified in Fig. 2. For instance, the system of Fig. 2a is built by moving tray section 3 from the Petlyuk column along with the condenser to the top of the first column. The resulting structure provides two liquid streams that flow unidirectionally from the first to the second column. This configuration is identified in this work as a fully coupled arrangement with liquid flows (FC-L). If one removes tray section 6 of the Petlyuk column and the reboiler and places them in the bottom of the first column, a fully coupled arrangement with unidirectional vapor flows from the first to the second column is obtained (FC-V, Fig. 2b). The other four new arrangements are obtained through a reduction in the number of interconnections of the original Petlyuk scheme; to accomplish this, the addition of a condenser or a reboiler in the prefractionator is required. Fig. $2 \mathrm{c}$ and d show two modifications to the original Petlyuk configuration with a reduction of one interconnecting stream that result in structures with vapor (MP-V) or with liquid (MP-L) interconnections. Partially coupled structures with vapor (PC-V) or with liquid (PC-L) interconnections are shown in Fig. 2e and f; such structures emerge from the elimination of one interconnection to the fully coupled arrangements of Fig. $2 a$ and $b$. Jiménez et al. (2003) have reported a study on the design and optimization of the operating conditions to achieve minimum energy consumption for the schemes of Fig. 2, and found that 


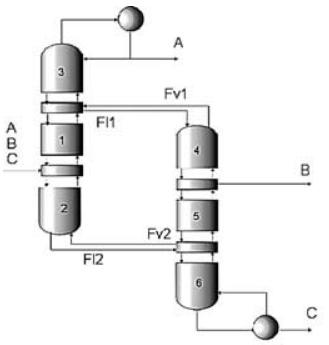

(a) FC-L

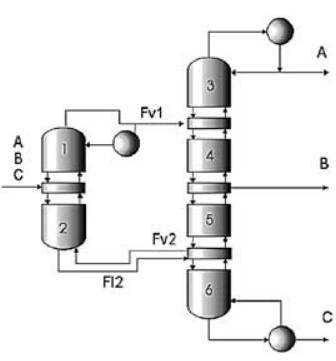

(c) MP-V

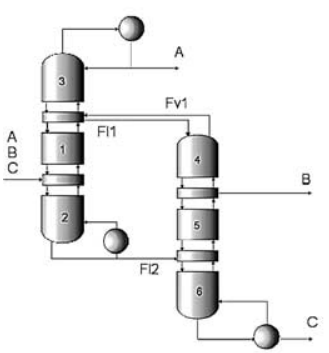

(e) PC-L

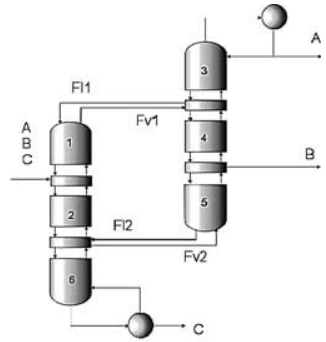

(b) FC-V

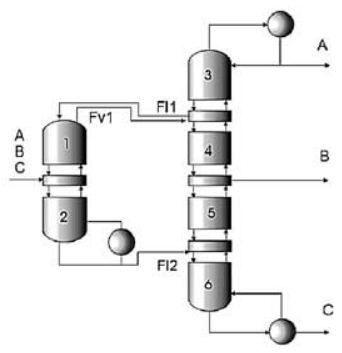

(d) MP-L

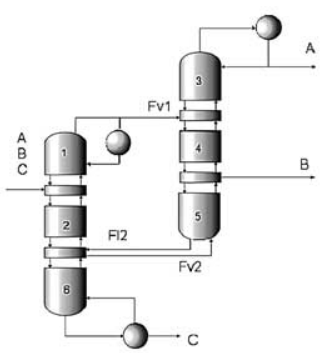

(f) PC-V
Fig. 2. Alternative schemes to the Petlyuk column.

such new systems can provide similar energy efficiencies as the original Petlyuk system. Although the simpler structures of the new designs seem to favor their dynamic properties, no previous analysis on this matter has been reported. The objective of this work is to conduct an initial analysis on the controllability properties of these arrangements.

\section{Design of the thermally coupled systems}

Before a dynamic analysis can be performed, a base design for the Petlyuk system and for each of the six alternative structures needs to be obtained. For the Petlyuk column, the method by Hernández and Jiménez (1999b) was used. The design procedure is as follows. A base design is obtained from the tray structure of a sequence of conventional distillation columns consisting of a prefractionator followed by two binary separations. After a section analogy procedure is used to provide the tray arrangement of the Petlyuk system, a search is conducted on the interconnecting streams to detect the values that provide the best energy efficiency for the system. A base design with minimum energy consumption is therefore detected.
The design (tray structure) of the Petlyuk system serves then as a basis to obtain the designs of the six alternative schemes. The corresponding tray structure analogy for each new arrangement is shown in Fig. 2 with respect to the sections of Fig. 1c. To provide a consistent comparison of the alternate schemes with the Petlyuk column, the remaining degrees of freedom for each arrangement were also used to detect the operating conditions that provided minimum energy consumption for each case; the details of the procedure are available in Jiménez et al. (2003).

\section{Dynamic analysis}

To carry out the comparative controllability analysis for the seven integrated sequences, a ternary mixture with hydrocarbon compounds was used. A feed stream of $n$-pentane (A), $n$-hexane (B) and $n$-heptane (C) with a low amount of the intermediate component (A, B, C equal to $0.4,0.2,0.4$ ) was considered. The feed composition reflects a typical value for which higher energy savings of the Pelyuk system have been reported. The feed flowrate was taken as $45.5 \mathrm{kmol} / \mathrm{h}$, and assumed to be as a saturated liquid. Design specifications for product compositions included $98.7 \%$ purity in A, $98 \%$ purity in $\mathrm{B}$ and $98.6 \%$ purity in $\mathrm{C}$. The columns of the conventional sequence (prefractionator and binary splits $\mathrm{A} / \mathrm{B}$ and $\mathrm{B} / \mathrm{C}$ ) that provide the tray structure for the Petlyuk system (Hernández \& Jiménez, 1999b) were designed assuming reflux ratios of 1.3 times the minimum values. The design pressure for any required column was chosen so that the condenser could be operated with cooling water (Jiménez et al., 2003).

The control analysis was conducted in two parts. First, a singular value decomposition (SVD) technique was used to obtain a comparative outlook on the theoretical control properties of the seven distillation structures. Then, closed loop control tests were run to complement the theoretical properties predicted by SVD. The closed loop runs were conducted under servo and load disturbance scenarios. The aim of the control analysis is to identify the best structures from a dynamic point of view, and to corroborate if the new arrangements indeed improve the dynamic characteristics of the Petlyuk configuration.

\section{Singular value decomposition}

Open loop dynamic responses to set point changes around the assumed operating point (which corresponded to that with minimum energy consumption for each configuration) were obtained. The responses were obtained through the use of Aspen Dynamics. Transfer function matrices $(\mathbf{G})$ were then collected for each case, and they were subjected to SVD:

$\mathbf{G}=\mathbf{V} \Sigma \mathbf{W}^{\mathrm{H}}$

where $\Sigma$ is the diag $\left(\sigma_{1}, \ldots, \sigma_{n}\right) ; \sigma_{i}$ the singular value of $\mathbf{G}=\lambda_{i}^{1 / 2}\left(\mathbf{G G}^{\mathrm{H}}\right) ; \mathbf{V}$ the $\left(v_{1}, v_{2}, \ldots\right)$ matrix of left singular 
vectors, and $\mathbf{W}$ is the $\left(w_{1}, w_{2}, \ldots\right)$ matrix of right singular vectors. Two parameters of interest are the minimum singular value, $\sigma_{*}$, and the ratio maximum to minimum singular values, or condition number:

$\gamma^{*}=\frac{\sigma^{*}}{\sigma_{*}}$

The minimum singular value is a measure of the invertibility of the system and represents a measure of the potential problems of the system under feedback control. The condition number reflects the sensitivity of the system under uncertainties in process parameters and modeling errors. These parameters provide a qualitative assessment of the theoretical control properties of the alternate designs. The systems with higher minimum singular values and lower condition numbers are expected to show the best dynamic performance under feedback control. Jiménez et al. (2001) have shown the application of the SVD technique to compare the con-

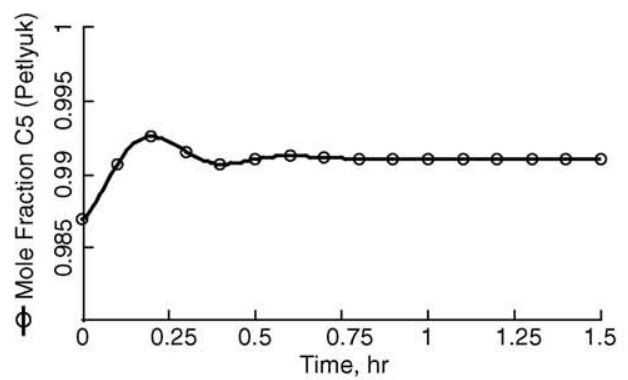

(a) Petlyuk

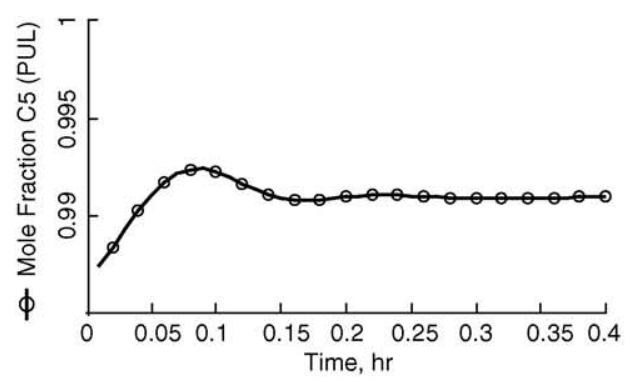

(b) FC-L

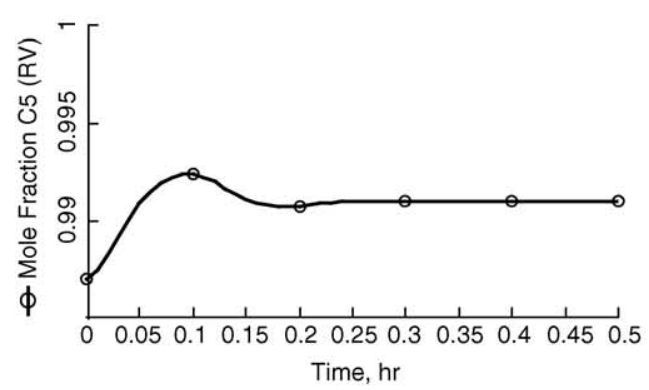

(d) MP-V

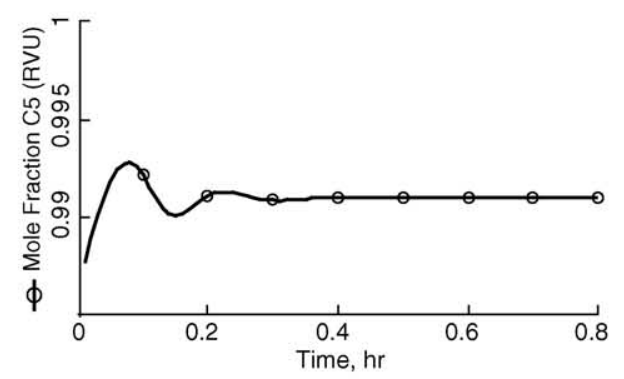

(f) PC-V

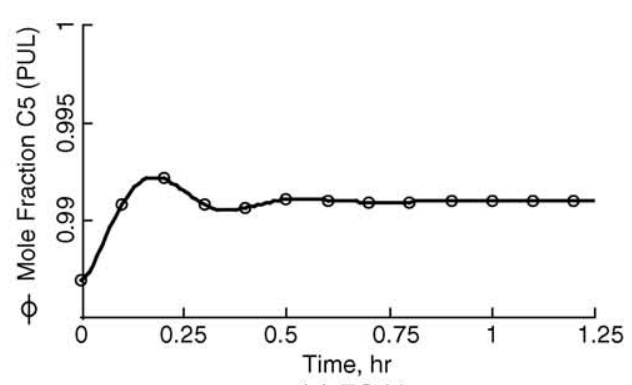

(c) FC-V

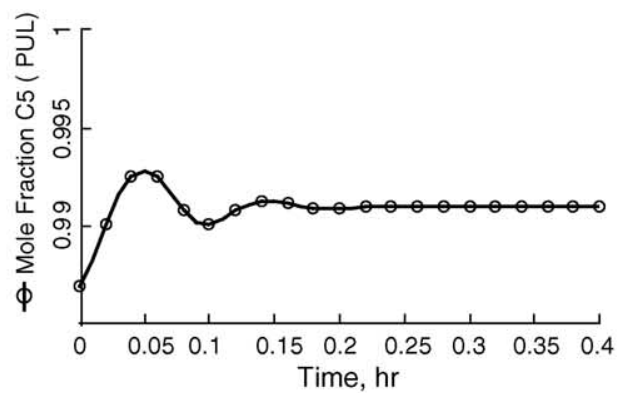

(e) MP-L

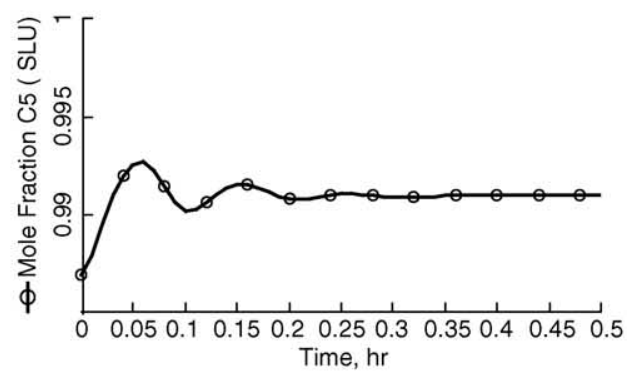

(g) PC-L

Fig. 3. Closed loop responses for a set point change in the light product (A) composition. 
trollability properties of the thermally coupled structures of Fig. 1 to those of sequences based on conventional distillation columns.

A full SVD analysis should cover a sufficiently complete range of frequencies. For this initial analysis of the alternative schemes to the Petlyuk configuration, we simply estimated the SVD properties for each separation system at zero frequency. Such analysis should give some preliminary in- dication on the control properties of each system around the nominal operating point.

Table 1 gives the results for the SVD test for each sequence. The fully coupled system with unidirectional liquid flows has the lowest value of the condition number, which implies that this sequence is better conditioned to the effect of disturbances than the other six integrated systems; such sequence also shows the highest value of the minimum sin-

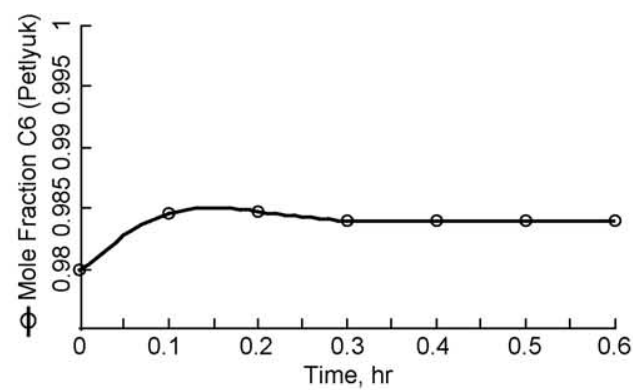

(a) Petlyuk

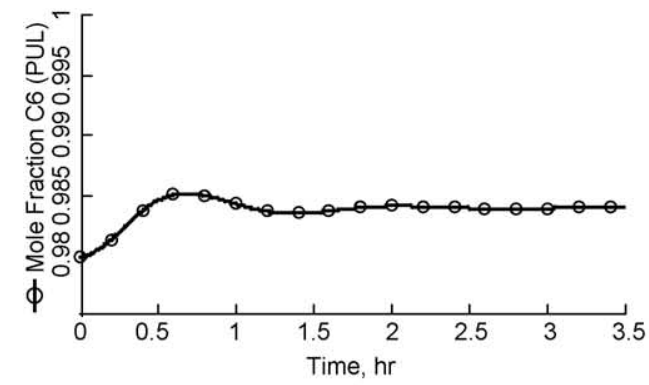

(b) FC-L

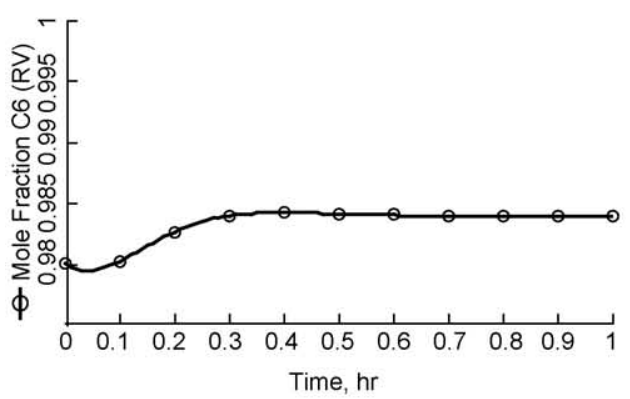

(d) MP-V

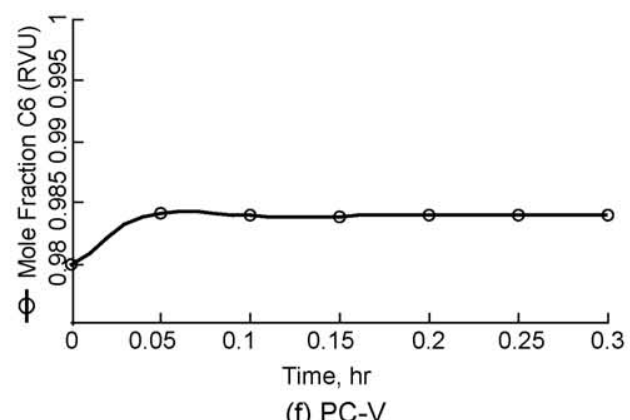

Fig. 4. Closed loop responses for a set point change in the intermediate product (B) composition.

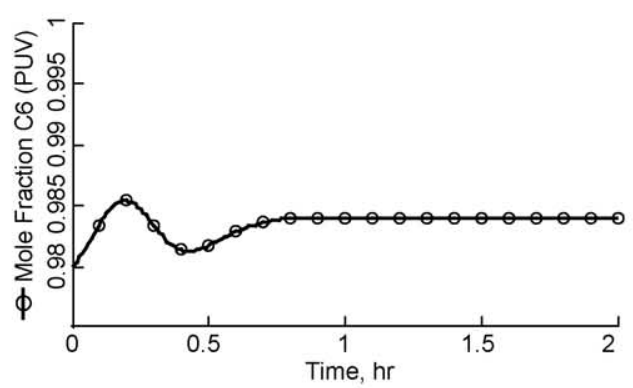

(c) FC-V

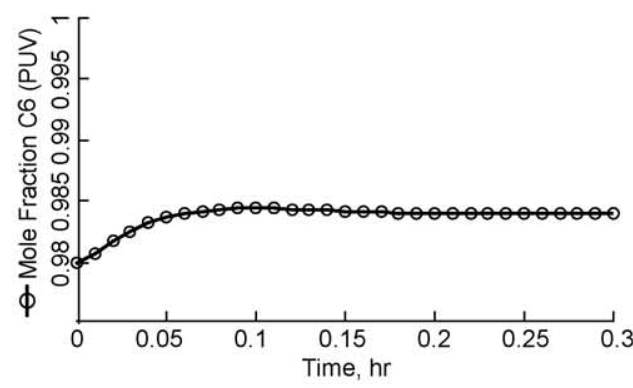

(e) MP-L

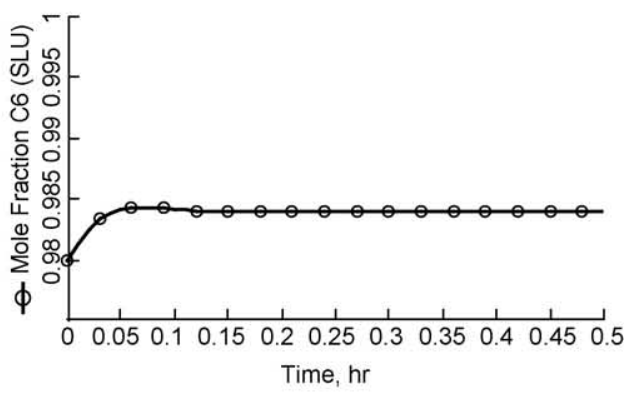

(g) PC-L 
gular value, which means that the FC-L column is expected to require lower control efforts under feedback operation than the other integrated sequences. On the other side of the analysis, the modified Petlyuk structure with liquid flows MP-L shows the worst values of the SVD parameters, which implies that, from the set of alternative structures, this sequence is worst conditioned to the effect of disturbances. Since the new structures were conceived to improve the control properties of the Petlyuk column, it should be highlighted that the Petlyuk configuration does not show the worst control properties of the seven sequences. The SVD parameters indicate that the theoretical properties of the modified Petlyuk struc-

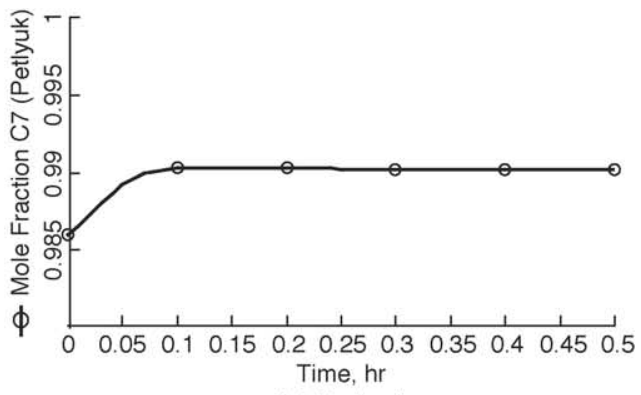

(a) Petlyuk

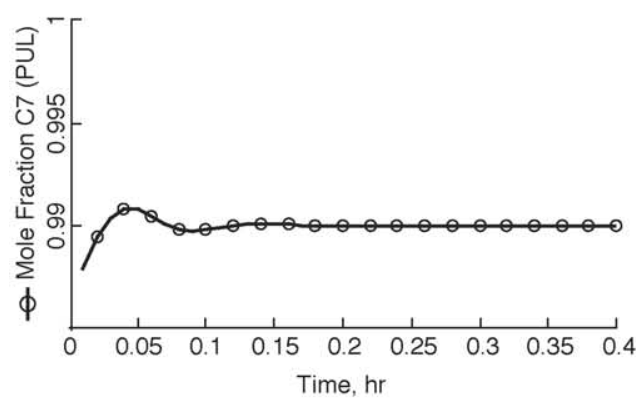

(b) FC-L

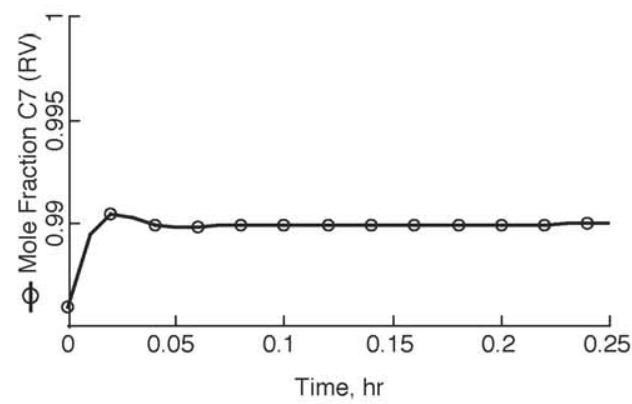

(d) MP-V

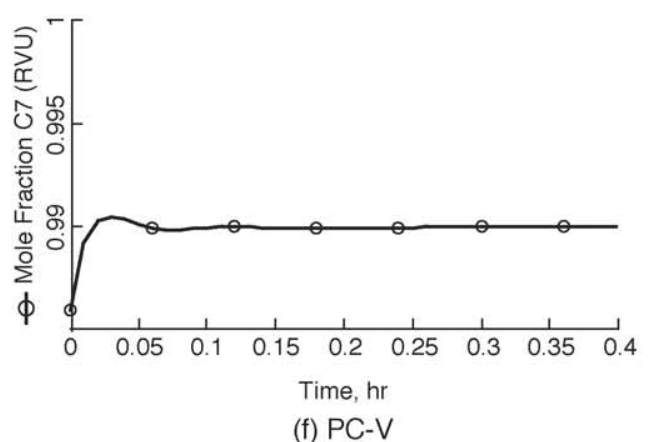

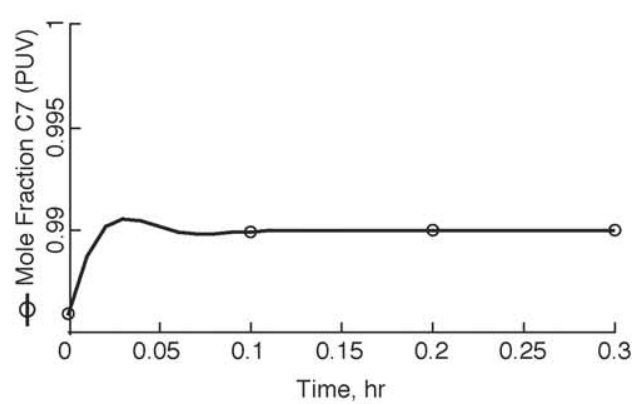

(c) FC-V

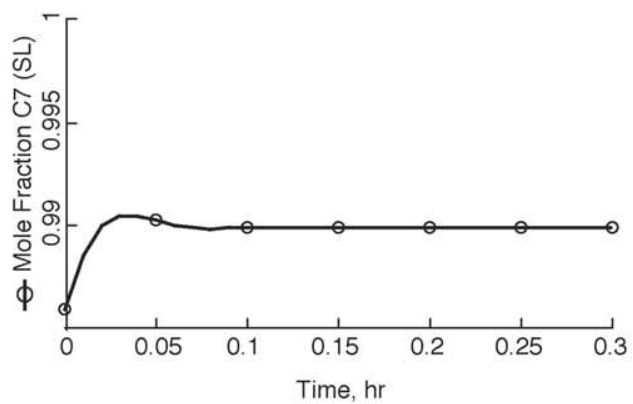

(d) MP-L

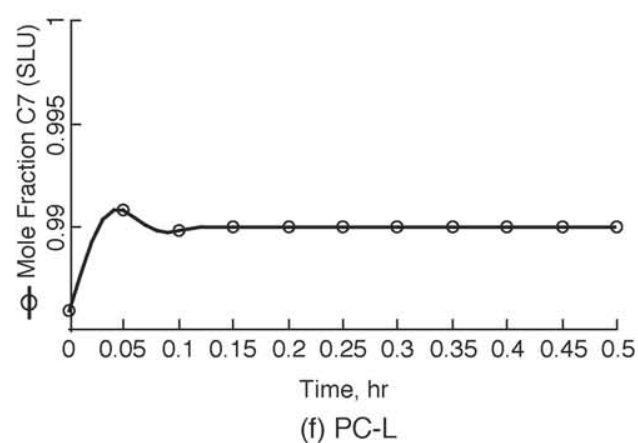

(f) PC-L

Fig. 5. Closed loop responses for a set point change in the heavy product (C) composition. 
tures (MP-V and MP-L), obtained through the elimination of one interconnecting stream, are expected to be worse than those of the original Petlyuk arrangement. Moreover, one can also notice that the reduction of one interconnection from the fully coupled unidirectional arrangements (FC-L and FC-V) produces the partially coupled arrangements (PC-L and PC$\mathrm{V})$ that deteriorate the theoretical controllability properties of the fully coupled structures. The best compromise on the theoretical controllability properties provided by the SVD ap- plication seems to be provided by the fully coupled structures FC-L and FC-V.

Overall, the results from this test indicate that the dynamic properties of the Petlyuk column may be improved by adjusting its design so that unidirectional flows are implemented. However, the reduction in the number of interconnections that provides simpler designs does not necessarily drive the expected operational advantages with respect to the original Petlyuk configuration.

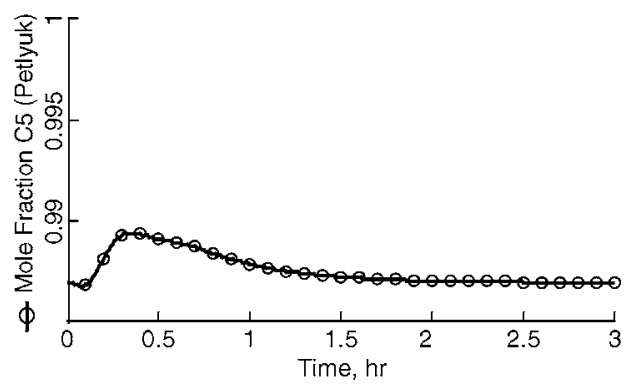

(a) Petlyuk

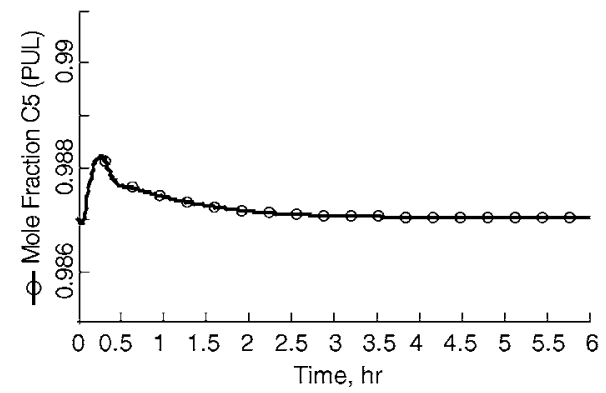

(b) FC-L

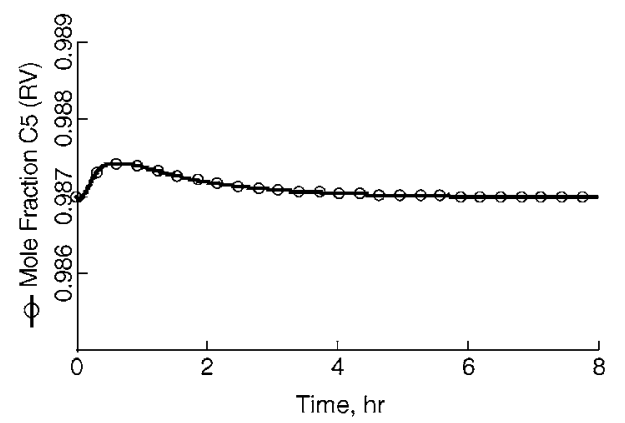

(d) MP-V

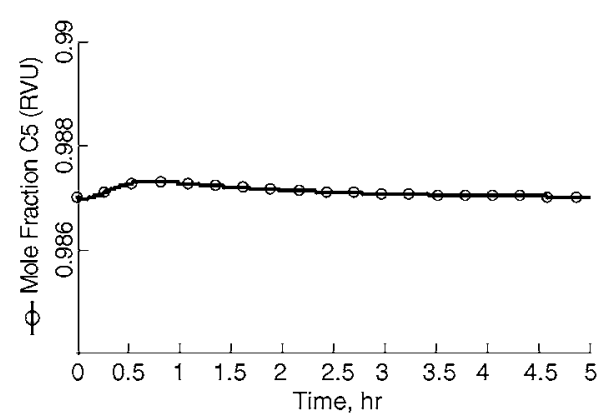

(f) PC-V

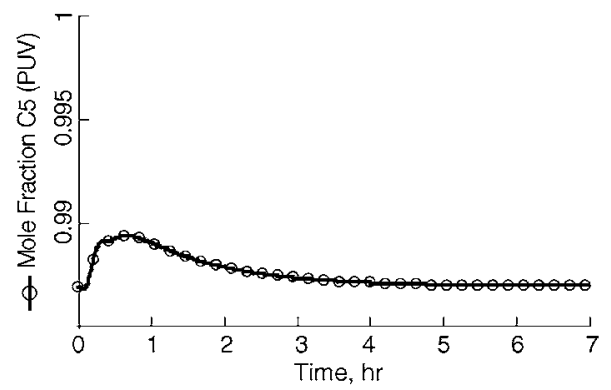

(c) FC-V

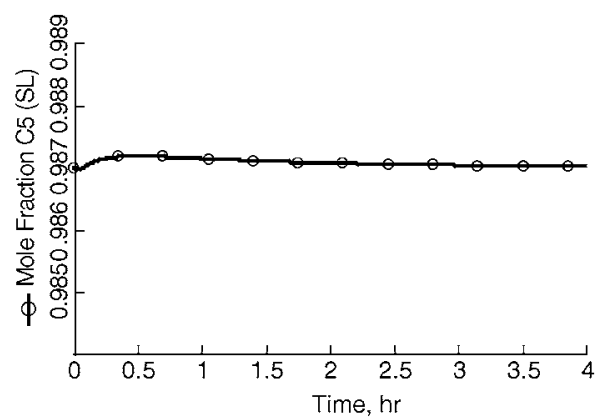

(e) MP-L

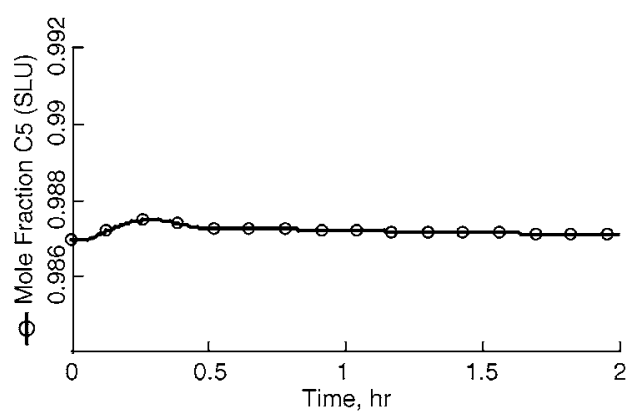

(g) PC-L

Fig. 6. Dynamic responses under a feed disturbance in the composition of the light (A) component. 


\section{Dynamic simulations}

The second part of the dynamic analysis called for some closed loop control tests. Two sets of dynamic simulations were carried out: (i) a step change was induced in the set point for each product composition under single-input, singleoutput (SISO) feedback control at each output flowrate and (ii) a 5\% change in the composition of one component (with a proportional adjustment in the composition of the other components to keep the same total feed flowrate) was implemented as a feed disturbance. The closed loop simulations were conducted with the aid of Aspen Dynamics. $K$ values for the hydrocarbon mixtures were estimated with the Chao-Seader equation (Seader \& Henley, 1998).

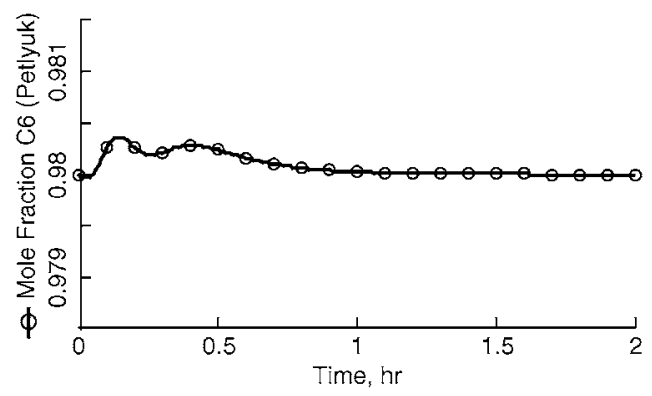

(a) Petlyuk

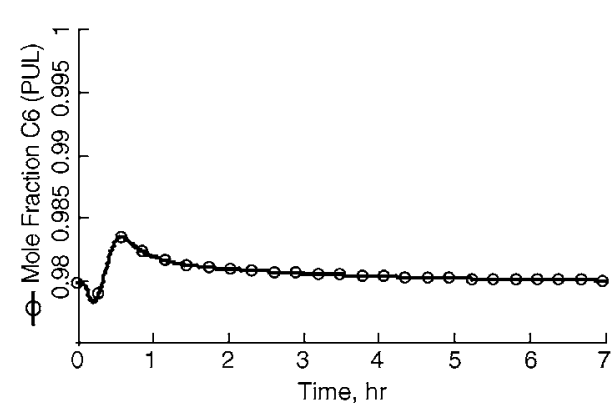

(b) FC-L

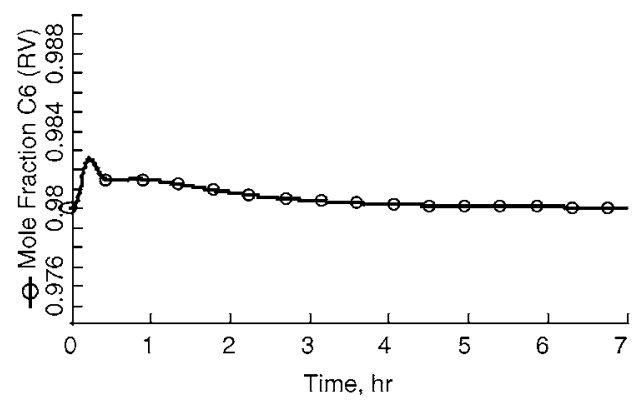

(d) MP-V

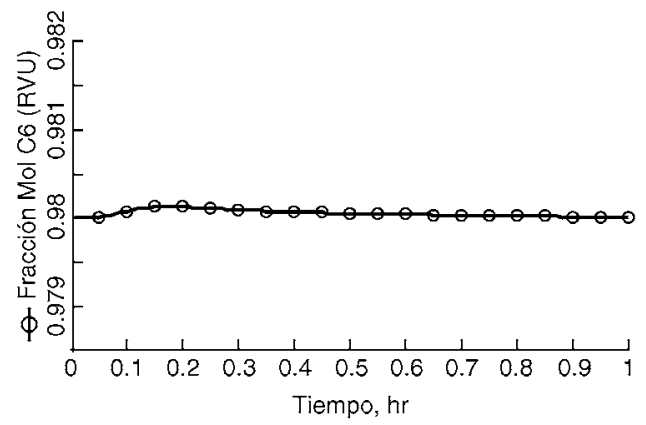

(f) PC-V

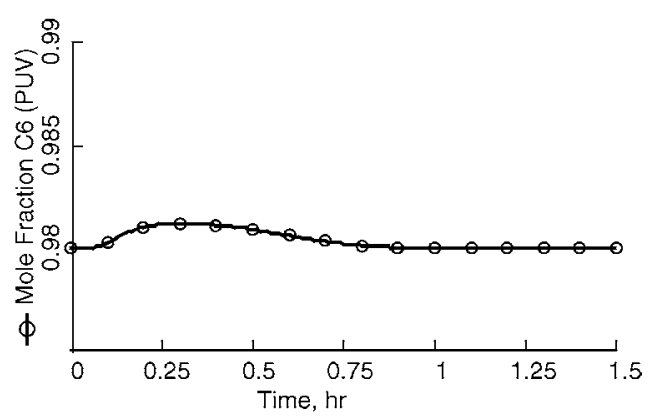

(c) FC-V

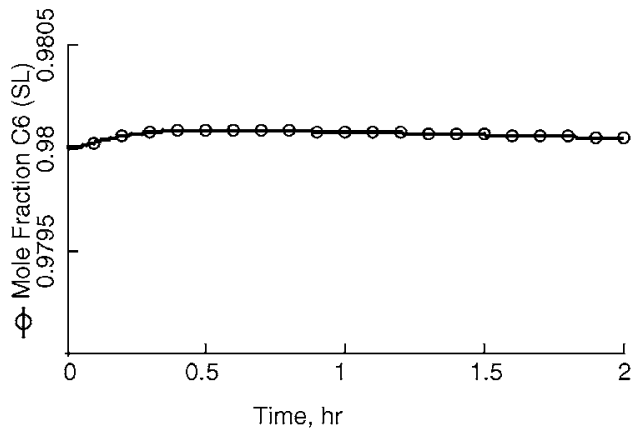

(e) MP-L

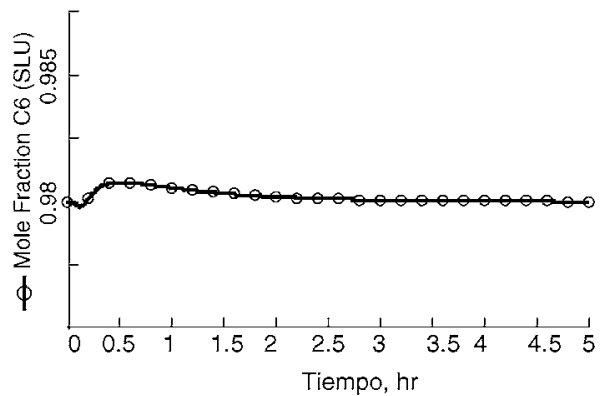

(g) PC-L

Fig. 7. Dynamic responses under a feed disturbance in the composition of the intermediate (B) component. 
The closed loop analysis should be conducted on a basis as consistent as possible. Therefore, the same type of controllers, the same tuning technique and the same control loops were used for each distillation configuration. For this initial comparative analysis, PI controllers were selected. The parameters of the controllers were tuned up through a minimization procedure of the integral of absolute error (IAE). For each control loop, aided by Aspen Dynamics, a search procedure was conducted on the gain constant and the integral time parameters until a minimum value of the IAE was obtained. The control loops used for the analysis were based on potential practical applications. The distillate stream, rich in A component, was controlled with the reflux flowrate, the side stream, which provides the intermediate product component, was controlled with the B product stream flowrate, and the composition of the bottoms stream that contains the

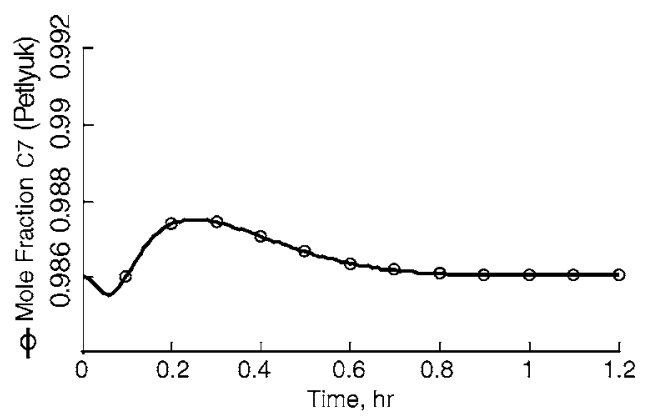

(a) Petlyuk

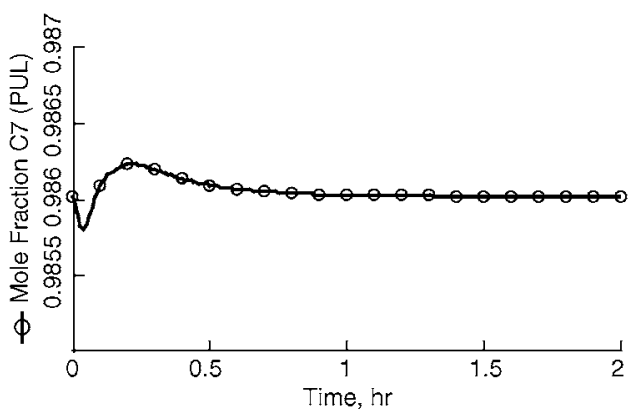

(a) FC-L

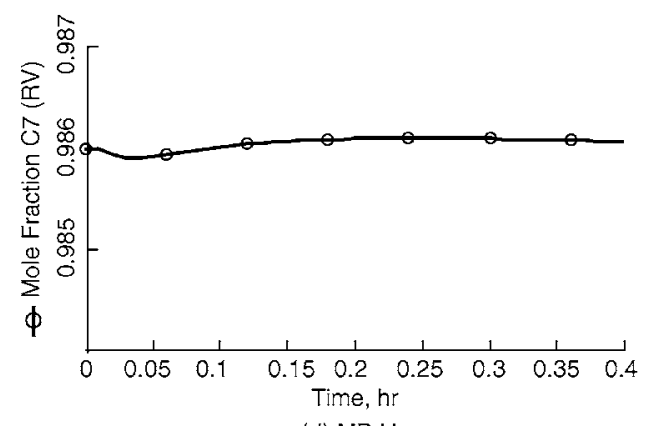

(d) MP-V

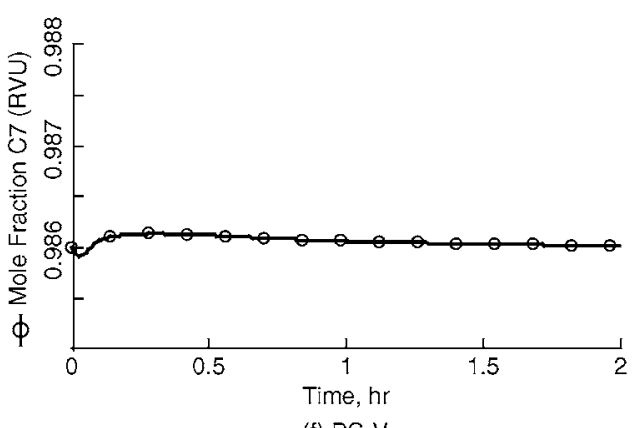

(f) PG-V

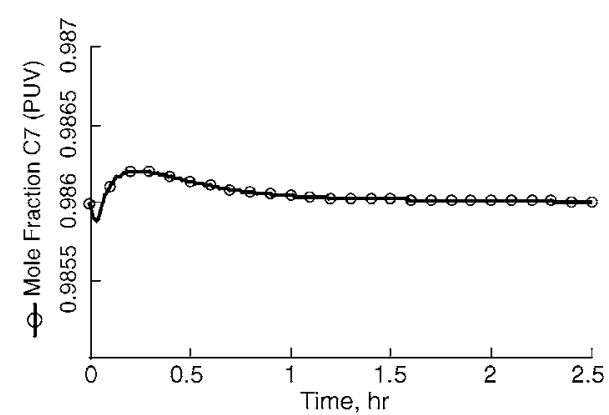

(b) FC-V

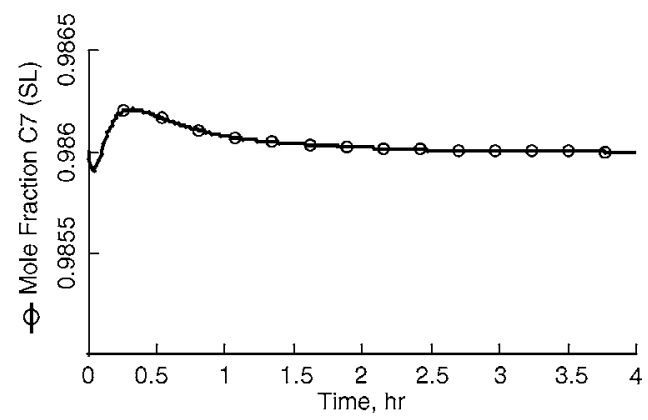

(e) MP-L

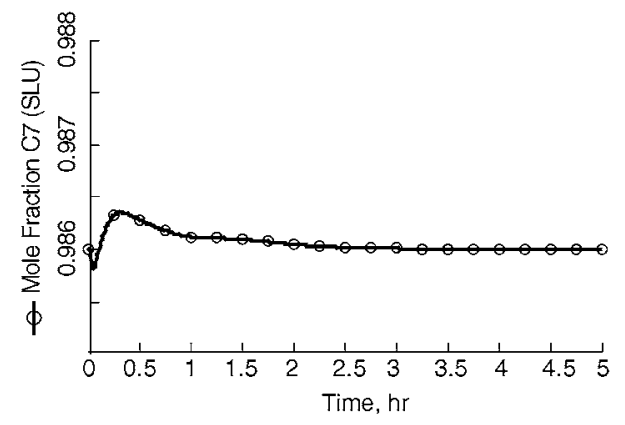

(g) PC-L

Fig. 8. Dynamic responses under a feed disturbance in the composition of the heavy (C) component. 
Table 1

Minimum singular value and condition number for each structure

\begin{tabular}{lll}
\hline Sequence & $\sigma_{*}$ & $\gamma^{*}$ \\
\hline Petlyuk & 0.0055 & 1260.47 \\
FC-L & $\mathbf{0 . 4 3 8 3}$ & $\mathbf{1 4 . 3 2 7 8}$ \\
FC-V & 0.0363 & 252.68 \\
MP-V & 0.0021 & 1684.21 \\
MP-L & $\mathbf{0 . 0 0 0 8}$ & $\mathbf{6 7 3 4 . 8}$ \\
PC-V & 0.0135 & 318.27 \\
PC-L & 0.0488 & 1054 \\
\hline
\end{tabular}

Boldface indicate the maximum and minimum values.

C component was controlled with the associated reboiler heat duty. The basis for the closed loop simulations regarding tuning methods and control loops are similar to the ones used earlier for the dynamic analysis of thermally coupled distillation systems by Segovia-Hernández et al. (2002, 2004).

\subsection{Set point changes}

The results from the individual servo tests are displayed in Figs. 3-5. All designs are able to reach the new values of product compositions imposed as step changes. From Fig. 3, one may notice that the fully coupled system FC-L and the modified Petlyuk structure MP-V provide the minimum settling time for the SISO control of the lightest component. The Petlyuk system and the fully coupled structure FC-V show the highest settling times, although the difference is not as significant with respect to the other options. For the intermediate component, the partially coupled structures PC-V and PC-L, and the modified Petlyuk arrangement MP-L provide fairly similar responses with a minimum settling time of about $0.05 \mathrm{~h}$, slightly better then the Petlyuk system. The fully coupled systems FC-L and FC-V show the worst responses with the highest settling times. From the step test for the heavy component, Fig. 5 shows that all systems provide suitable responses, with the modified Petlyuk configuration $\mathrm{MP}-\mathrm{V}$ and the partially coupled structure PC-V giving the minimum settling times.

Settling times can be used as a guide to screen alternatives, but a more formal quantification of the dynamic responses can be used to establish the superiority of a given separation structure. To provide such a comparison of the servo responses, IAE values were evaluated for each dynamic response, with the results given in Table 2. One can observe that the fully coupled column FC-L shows the best behav-

Table 2

IAE values for the set point tracking test

\begin{tabular}{llll}
\hline Sequence & $\mathrm{A}$ & $\mathrm{B}$ & $\mathrm{C}$ \\
\hline Petlyuk & $5.15 \times 10^{-4}$ & $2.87 \times 10^{-4}$ & $2.35 \times 10^{-4}$ \\
FC-L & $1.15 \times 10^{-4}$ & 0.0017 & $4.20 \times 10^{-5}$ \\
FC-V & $2.02 \times 10^{-4}$ & 0.0011 & $6.10 \times 10^{-5}$ \\
MP-V & $2.15 \times 10^{-4}$ & $3.31 \times 10^{-4}$ & $8.60 \times 10^{-4}$ \\
MP-L & $5.55 \times 10^{-4}$ & $7.77 \times 10^{-4}$ & $9.20 \times 10^{-4}$ \\
PC-V & $2.53 \times 10^{-4}$ & $9.91 \times 10^{-4}$ & $4.95 \times 10^{-4}$ \\
PC-L & $1.83 \times 10^{-4}$ & $8.57 \times 10^{-4}$ & $8.39 \times 10^{-4}$ \\
\hline
\end{tabular}

Table 3

IAE values for feed disturbance rejection analysis

\begin{tabular}{llll}
\hline Sequence & $\mathrm{A}$ & $\mathrm{B}$ & $\mathrm{C}$ \\
\hline Petlyuk & 0.0018 & $2.74 \times 10^{-5}$ & $5.83 \times 10^{-4}$ \\
FC-L & $3.63 \times 10^{-4}$ & 0.001104 & $1.50 \times 10^{-4}$ \\
FC-V & 0.0011 & 0.001768 & $2.20 \times 10^{-4}$ \\
MP-V & $8.32 \times 10^{-4}$ & 0.003323 & $4.21 \times 10^{-4}$ \\
MP-L & 0.0063 & $2.87 \times 10^{-4}$ & $6.10 \times 10^{-4}$ \\
PC-V & $6.45 \times 10^{-4}$ & $7.27 \times 10^{-5}$ & $1.75 \times 10^{-4}$ \\
PC-L & $5.58 \times 10^{-4}$ & $8.58 \times 10^{-5}$ & $6.09 \times 10^{-4}$ \\
\hline
\end{tabular}

ior (smallest values of IAE) for the control of the light and the heavy components, while the modified Petlyuk sequence MP-L exhibits the worst dynamic behavior. These results are consistent with the predictions given by SVD. The closed loop results for the intermediate component, however, deviate from the predictions of the SVD technique. In such case, the Petlyuk column shows unexpectedly the best IAE value.

\subsection{Feed disturbance rejection}

The results for the feed disturbance scenarios are displayed in Figs. 6-8, with the IAE values for each case summarized in Table 3. It can be observed that the fully coupled sequence FC-L shows the best behavior for the adjustment of the composition of the extreme components $\mathrm{A}$ and $\mathrm{C}$, with fast responses and with no overshoot problems, while the modified Petlyuk structure MP-L shows the worst responses for the control of these components among the seven sequences under comparison, reflected in high settling times and the highest IAE values. As in the case of the servo tests, the dynamic responses for the disturbance rejection runs can be collected into two groups. One of them corresponds to the control of the extreme components, which are quite similar in the shape of their dynamic responses and consistent with the results provided by SVD, and the other case corresponds to the different behavior of the closed loop responses for the intermediate component. Interestingly, the Petlyuk column shows again the best behavior for the latter case; as reflected in Fig. 7 and by the IAE values, the implementation of the PI controllers on the Petlyuk system provides a better response for the composition adjustment of the intermediate component under the implemented feed disturbance with respect to the dynamic behavior of the alternative schemes.

\section{Conclusions}

A comparative analysis on the theoretical control properties and closed loop responses between the Petlyuk column and six alternative schemes has been presented. The set of alternative structures emerges from a reduction in the number of interconnections or by correcting the bidirectional nature of the interconnections of the Petlyuk original design. The results from the theoretical control properties evaluated 
from the singular value decomposition method indicate that the major disadvantage of the Petlyuk column seems to be mainly due to the bidirectionality of the two interconnecting vapor streams. Thus, alternative systems with two interconnections but with unidirectional interconnecting flows may improve the controllability properties of the Petlyuk system. On the other hand, the SVD results show that a reduction in the number of interconnections does not necessarily provide the operational advantages originally expected from a modified and simpler structural design. SISO closed loop control tests were also conducted, and in general the dynamic simulations supported the predictions of the SVD technique for the individual control of the extreme components of the ternary mixture. For the control of the intermediate component, however, the Petlyuk column showed unexpectedly the best dynamic performance. Although multivariable control tests need to be carried out, and the effect of feed composition and feed mixture properties remain to be explored, two major conclusions can be addressed from this initial study. One, the theoretical controllability properties of the Petlyuk column were not improved by all of the alternative sequences that were conceptually designed to provide simpler and more controllable structures; furthermore, from all the set of alternatives, the Petlyuk column showed a superior dynamic performance for the SISO control of the intermediate component. Second, we have found that the properties of the Petlyuk configuration can be improved through the correction of the bidirectionality of its interconnecting streams, but can be deteriorated by the reduction in the number of interconnections.

\section{References}

Abdul Mutalib, M. I., \& Smith, R. (1998). Operation and control of dividing wall distillation columns. Part 1. Degrees of freedom and dynamic simulation. Transactions of IChemE, 76(Part A), 308.

Agrawal, R., \& Fidkowski, Z. T. (1998). More operable arrangements of fully thermally coupled distillation columns. AIChE Journal, 44(11), 2565 .
Agrawal, R., \& Fidkowski, Z. T. (1999). New thermally coupled schemes for ternary distillation. AIChE Journal, 45(3), 485.

Dünnebier, G., \& Pantelides, C. (1999). Optimal design of thermally coupled distillation columns. Industrial and Engineering Chemistry Research, 38, 162.

Fidkowski, Z., \& Krolikowski, L. (1990). Energy requirements of nonconventional distillation systems. AIChE Journal, 36, 1275.

Flores, O. A., Cárdenas, J. C., Hernández, S., \& Rico-Ramírez, V. (2003). Thermodynamic analysis of thermally coupled distillation sequences. Industrial and Engineering Chemistry Research, 42, 5940.

Glinos, K., \& Malone, F. (1988). Optimality regions for complex column arrangements for ideal distillation. Chemistry Engineering Research and Design, 66, 229.

Hernández, S., \& Jiménez, A. (1999a). Controllability analysis of thermally coupled distillation systems. Industrial and Engineering Chemistry Research, 38, 3957.

Hernández, S., \& Jiménez, A. (1999b). Design of energy-efficient Petlyuk systems. Computers and Chemical Engineering, 23, 1005.

Jiménez, A., Hernández, S., Montoy, F. A., \& Zavala-García, M. (2001). Analysis of control properties of conventional and nonconventional distillation sequences. Industrial and Engineering Chemistry Research, 40, 3757.

Jiménez, A., Ramírez, N., Castro, A., \& Hernández, S. (2003). Design and energy performance of alternative schemes to the Petlyuk distillation systems. Transactions of IChemE, 81(Part A), 518.

Rong, B. G., \& Kraslawski, A. (2003). Partially thermally coupled distillation systems for multicomponent separations. AIChE Journal, 49, 1340.

Seader, J. D., \& Henley, E. (1998). Separation process principles. New York: Wiley.

Segovia-Hernández, J. G., Hernández, S., \& Jiménez, A. (2002). Control behaviour of thermally coupled distillation sequences. Transactions of IChemE, 80(Part A), 783.

Segovia-Hernández, J. G., Hernández, S., Rico-Ramírez, V., \& Jiménez, A. (2004). A comparison of the feedback control behavior between thermally coupled and conventional distillation schemes. Computers and Chemical Engineering, 28, 811.

Tedder, D. W., \& Rudd, D. F. (1978). Parametric studies in industrial distillation. Part I. Design comparisons. AIChE Journal, 24, 303

Wolff, E. A., \& Skogestad, S. (1995). Operation of integrated threeproduct (Petlyuk) distillation columns. Industrial and Engineering Chemistry Research, 34, 2094.

Yeomans, H., \& Grossmann, I. E. (2000). Optimal design of complex distillation columns using rigorous tray-by-tray disjunctive programming models. Industrial and Engineering Chemistry Research, 39, 4326. 